\title{
Perfil de pessoas com sintomas depressivos e comportamento suicida em população geral de cidade mineira
}

\author{
Profile of people with depressive symptoms and suicide behavior in a general population in the \\ mining city
}

Perfil de personas con síntomas depresivos y comportamiento suicidio en una población general de la ciudad minera

\section{Resumo}

Objetivo: Analisar perfil sociodemografico de indivíduos com sintomatologia depressiva e comportamento suicida em população geral adulta. Método: Estudo exploratório-descritivo, quantitativo. Entrevistas com aplicação de questionário e escalas psicométricas para depressão em população geral adulta de Poços de Caldas/MG. Resultados: De 200 participantes, $73(36,5 \%)$ apresentaram sintomatologia depressiva, $62(84,92 \%)$ com depressão previamente diagnosticada, $27(37,0 \%)$ intensidade moderada, $11(15,1 \%)$ grave; $57(78,1 \%)$ mulheres, $22(30,1)$ entre 29-39 anos, $67(91,78 \%)$ brancos, $47(64,4 \%)$ católicos, $39(53,42 \%)$ casados, $32(43,8 \%)$ com até 02 filhos, $20(27,39 \%)$ ensino fundamental incompleto, $51(69,86 \%)$ empregados, $34(46,58 \%)$ renda individual até 02 salários mínimos; 39 (53,43\%) viviam com companheira/marido/esposa/filhos; 23 (31,50\%) moravam em área de vulnerabilidade social; $40(54,8 \%)$ apresentaram comorbidades clínicas; $30(41,1 \%)$ com tempo diagnóstico de depressão entre 0 a 5 anos; 54 $(73,97 \%)$ referiram tratamento, dos quais $36(66,7 \%)$ de 0 a 5 anos, $10(18,5 \%)$ entre 12 a 17 anos; $26(35,61 \%)$ com ideação suicida, sendo $13(50,0 \%)$ nos últimos 5 anos, $11(42,3 \%)$ não referiram tratamento, e dos $15(57,7 \%)$ que referiram tratamento, $06(40 \%)$ o realizou nos últimos 03 meses, sendo $07(46,7 \%)$ apenas medicamentoso e 03 $(11,5 \%)$ o tratamento foi hospitais psiquiátricos. Conclusão: Prevalência de sintomatologia depressiva na população do estudo $(36,5 \%)$ foi maior que média nacional, com prevalência de intensidade dos sintomas moderado/grave. Participantes sem sintomatologia prévia apresentaram maior severidade dos sintomas depressivos, sendo mulheres, desempregadas, jovens, baixa escolaridade e baixa renda, com familiar ou a mesma com transtorno mental, usuário de álcool/drogas ou doenças crônicas.

Palavras-chave: Depressão; Comportamento suicida; Epidemiologia; Saúde mental.

\begin{abstract}
Objective: To analyze the sociodemographic profile of individuals with depressive symptoms and suicidal behavior in a general adult population. Method: Exploratory-descriptive, quantitative study. Interviews with questionnaires and psychometric scales for depression in a general adult population in Poços de Caldas / MG. Results: Of 200 participants, $73(36.5 \%)$ had depressive symptoms, $62(84.92 \%)$ with previously diagnosed depression, $27(37.0 \%)$ moderate intensity, $11(15.1 \%)$ severe; 57 (78.1\%) women, $22(30.1)$ between 29-39 years, 67 (91.78\%) white, 47 $(64.4 \%)$ Catholics, $39(53,42 \%)$ married, 32 ( $43.8 \%)$ with up to 02 children, $20(27.39 \%)$ incomplete primary education, $51(69.86 \%)$ employed, 34 (46.58\%) individual income up to 02 minimum wages; 39 (53.43\%) lived with a partner / husband / wife / children; 23 (31.50\%) lived in an area of social vulnerability; 40 (54.8\%) had clinical comorbidities; $30(41.1 \%)$ with time since diagnosis of depression between 0 and 5 years; $54(73.97 \%)$ reported treatment, of which $36(66.7 \%)$ between 0 and 5 years old, $10(18.5 \%)$ between 12 and 17 years old; $26(35.61 \%)$ with suicidal ideation, $13(50.0 \%)$ in the last 5 years, $11(42.3 \%)$ did not report treatment, and of the $15(57.7 \%)$ who reported treatment, 06 ( 40\%) performed it in the last 03 months, with 07 (46.7\%) only on medication and $03(11.5 \%)$ the treatment was psychiatric hospitals. Conclusion: Prevalence of depressive symptoms in the study population $(36.5 \%)$ was higher than the national average, with moderate / severe prevalence of symptom intensity. Participants with no previous symptoms presented greater severity of depressive symptoms, being women, unemployed, young
\end{abstract}


people, low education and low income, with family members or the same with mental disorders, alcohol / drug users or chronic diseases.

Keywords: Depression; Suicidal behavior; Epidemiology; Mental health.

\begin{abstract}
Resumen
Objetivo: Analizar el perfil sociodemográfico de individuos con síntomas depresivos y conducta suicida en una población adulta general. Método: Estudio exploratorio-descriptivo, cuantitativo. Entrevistas con cuestionarios y escalas psicométricas para la depresión en una población general adulta de Poços de Caldas / MG. Resultados: De 200 participantes, $73(36,5 \%)$ tenían síntomas depresivos, $62(84,92 \%)$ con depresión previamente diagnosticada, 27 $(37,0 \%)$ de intensidad moderada, $11(15,1 \%)$ grave; $57(78,1 \%)$ mujeres, $22(30,1)$ entre 29-39 años, $67(91,78 \%)$ blancas, $47(64,4 \%)$ católicas, $39(53,42 \%)$ casadas, 32 ( 43,8\%) con hasta 02 hijos, $20(27,39 \%)$ educación primaria incompleta, $51(69,86 \%)$ ocupados, 34 (46,58\%) ingresos individuales hasta 02 salarios mínimos; 39 (53,43\%) vivían con pareja / marido / mujer / hijos; 23 (31,50\%) vivían en un área de vulnerabilidad social; 40 (54,8\%) tenían comorbilidades clínicas; $30(41,1 \%)$ con tiempo desde el diagnóstico de depresión entre 0 y 5 años; $54(73,97 \%)$ refirieron tratamiento, de los cuales $36(66,7 \%)$ entre 0 y 5 años, $10(18,5 \%)$ entre 12 y 17 años; $26(35,61 \%)$ con ideación suicida, $13(50,0 \%)$ en los últimos 5 años, $11(42,3 \%)$ no informaron tratamiento y de los $15(57,7 \%)$ que informaron tratamiento, 06 ( 40\%) lo realizó en los últimos 03 meses, 07 (46,7\%) solo con medicación y 03 (11,5\%) el tratamiento fue en hospitales psiquiátricos. Conclusión: La prevalencia de síntomas depresivos en la población de estudio $(36,5 \%)$ fue superior a la media nacional, con una prevalencia moderada / grave de la intensidad de los síntomas. Los participantes sin síntomas previos presentaron mayor gravedad de síntomas depresivos, siendo mujeres, desempleados, jóvenes, de baja escolaridad y bajos ingresos, con familiares o lo mismo con trastornos mentales, consumidores de alcohol / drogas o enfermedades crónicas.
\end{abstract}

Palabras clave: Depresión; Comportamiento suicida; Epidemiología; Salud mental.

\title{
1. Introdução
}

Segundo as estimativas da Organização mundial de Saúde (OMS), cerca de 350 milhões de pessoas sofrem de depressão no mundo, tornando assim a depressão um problema grave de saúde publica (WHO, 2019), sendo que as projeções para 2030 apontam a depressão o mal mais prevalente do planeta e a primeira causa de incapacidades e a segunda maior causa de anos vividos com incapacidade no mundo (WHO, 2018). Estima-se que 50\% dos doentes em todo o planeta não recebem o tratamento adequado e tal fato gere quadros severos de absenteísmo e invalidez para o trabalho, alteração das relações sociais e comportamento suicida (WHO, 2019).

A falha em detectar indivíduos depressivos em níveis leves e moderados favorece o agravamento da depressão, com efeitos importantes sobre a capacidade para estudar, trabalhar e ter boa qualidade de vida (Munhoz, 2012). A detecção de casos de depressão tem sido eficaz para diminuir o número de consultas subsequentes, encurtar a duração do episódio depressivo e atenuar os efeitos da depressão em longo prazo (Güngör, Akgül, Taymur, Demirci \& İnel, 2020).

Relatório da OMS de 2014, corroborado em 2019, indicou que aproximadamente 804 mil pessoas cometeram suicídio em 2012 e uma tentativa prévia de suicídio é o mais importante fator de risco a ser observado - independente de diagnóstico de transtorno mental (WHO, 2014; WHO, 2019).

Quanto ao comportamento suicida este por sua vez engloba pensamentos de autodestruição, autoagressão, manifestada por gestos suicidas e tentativas de suicídio, e, finalmente, o próprio suicídio (WHO, 2019), tendo etiologia multifatorial, porém sendo a depressão importante fator predisponente e estima-se que $17 \%$ dos pacientes com depressão clínica grave tentam o suicídio pelo menos uma vez (APA, 2015).

O índice global anual de suicídios, padronizado por idade, mostrou incidência de $11,4 \%$ (15 homens e 08 mulheres por cem mil habitantes). Globalmente, suicídios representam $50 \%$ de todos os casos de morte violenta entre homens e $71 \%$ entre mulheres. Quanto à idade, taxas de suicídio são mais elevadas entre pessoas com idade igual ou superior a 70 anos (ambos os gêneros) na maioria das regiões do mundo. Mas em alguns países as taxas de suicídio são maiores entre os jovens e, globalmente, o suicídio é a segunda principal causa de morte entre jovens de 15 a 29 anos de idade - com $75 \%$ dos casos ocorridos em países de baixa ou média renda per capita (WHO, 2019). 
O suicídio geralmente é o desfecho trágico de transtornos psiquiátricos como os transtornos afetivos, transtornos psicóticos e uso de substâncias psicoativas. Em quase 90\% dos casos de suicídio há o diagnóstico de transtorno mental ou de uso abusivo de substâncias psicoativas (WHO, 2018).

Desta forma, o objetivo deste estudo foi analisar perfil sociodemográfico de indivíduos com sintomatologia depressiva e comportamento suicida em população geral adulta.

\section{Metodologia}

\subsection{Aspectos éticos}

Garantiu-se respeito às normas que regulamentam a pesquisa com seres humanos. A pesquisa deu-se após aprovação do Comitê de Ética em Pesquisa da Universidade Federal de São Paulo, sob parecer nº 1.813.770/2016. Os participantes consentiram em participar e foi aposto no Termo de Consentimento Livre e Esclarecido.

\subsection{Tipo de estudo}

Estudo descritivo-exploratório, com abordagem quantitativa (Marconi \& Lakatos, 2005), realizado em Poços de Caldas/MG. Período da coleta de dados se deu entre janeiro de 2017 a outubro de 2018.

\subsection{Procedimentos metodológicos}

Durante o primeiro contato com o participante, foi explicado o que compreendia a pesquisa, foram resolvidas as dúvidas, e após consentimento em participar, foi realizada entrevista em data, local e horário aceito pelo mesmo.

A população foi de residentes e domiciliados na cidade de Poços de Caldas/MG. A amostra probabilística e aleatória estratificada foi definida por conveniência a 200 indivíduos, escolhida aleatoriamente entre o centro e os três pontos cardeais da cidade (Sul, Leste e Oeste), mantida a proporção de 50 indivíduos por área geográfica. A exclusão da zona norte se deu ao fato desta ser área de mata preservada no Município e não possuir habitações. A quantidade de entrevistas foi dividida pela quantidade de bairros das regiões para que houvesse divisão igualitária entre a quantidade de entrevistados por bairro. Houve sorteio da rua de cada bairro e a coleta teve início pelo número de residência mais baixo da rua; após, a abordagem foi realizada do outro lado da rua, respeitada a alternância de duas casas entre um entrevistado e outro. Tal cuidado se fez necessário a fim de evitar comunicação entre os vizinhos e contaminação das respostas dos participantes.

Os critérios de inclusão foram: indivíduos de ambos os sexos; com idade acima de 18 anos; residentes e domiciliados em Poços de Caldas/MG em tempo igual ou superior a doze meses e com condições cognitivas para participar do estudo. Não houve exclusão.

\subsection{Coleta e organização dos dados}

Os instrumentos para coleta de dados utilizados foram escalas psicométricas para sintomatologia depressiva, aplicadas na seguinte ordem: Inventário de Depressão de Beck, Escala de Avaliação para Depressão de Hamilton e Escala de Avaliação para Depressão de Montgomery - Asberg. Para essas escalas, foi solicitado ao participante que respondesse para cada item qual alternativa descrevia melhor como se sentia na última semana, incluído o dia da pesquisa. A seguir, realizou-se a entrevista por meio de questionário semiestruturado confeccionado pelos pesquisadores com dados referentes a sexo, idade, bairro e região de moradia, cor/raça, religião, estado civil, escolaridade, quantidade de pessoas que residem no domicílio e atividade laboral; dados a respeito do histórico de doença na família, histórico pessoal, antecedentes de depressão e comportamento suicida. 


\subsection{Análise dos dados}

Realizadas de acordo com os escores estabelecidos para cada escala psicométrica e tratamento estatístico dos resultados de cada escala com as variáveis sociodemográficas por meio de testes. Para comparação de proporções entre grupos, foi aplicado o Teste Qui-Quadrado ou Teste Exato de Fisher, quando indicado. Foram traçadas correlações lineares entre os três instrumentos de avaliação da depressão segundo o método de Pearson. Foram considerados como estatisticamente significativos resultados com probabilidade de erro do tipo I inferior a 5,00\% ( $\mathrm{p}<0,005)$. Os dados foram tabulados com auxílio do pacote Microsoft Office 2010 e analisados com o pacote estatístico SPSS (Statistical Package Social Science), versão 20.0.

\section{Resultados}

Dos 200 participantes, o perfil sociodemográfico evidenciou maioria composta pelo gênero feminino (61\% $\mathrm{p}<0,001$ ), idade entre 29 a 39 anos (26\%), branco (84\%), católico (69\%), casado (62\%), com filhos (76,5\% - p<0,001), ter dois filhos $(46 \%)$, ensino fundamental incompleto $(29 \%$ - $\mathrm{p}<0,001)$ e ensino médio completo $(20 \%$ - $\mathrm{p}<0,001)$, com até 3 pessoas no domicilio (26,5\%), a viver com companheiros/filhos/cônjuges (62\%), exercia atividade laboral não remunerada $(25,5 \%)$, aposentados $(18,5 \%)$ e desempregados $(11,5 \%$ - p<0,001), com renda individual de até 2 salários mínimos $(37,5 \%$ - p<0,001) e 5 salários mínimos ((38,0\%-p p 0,001), renda familiar de 2 a 5 salários mínimos $(50,5 \%)$, uso de redes sociais como facebook $(88,5 \%$ - p $<0,001)$ e como recreação estar com os amigos $(36 \%)$. As variáveis com significância estatística estão relacionadas a presença de sintomatologia depressiva.

Na Tabela 1 encontram-se os dados quanto à presença e intensidade de sintomas depressivos pela aplicação das escalas para participantes com e sem diagnóstico prévio.

Tabela 1. Presença e intensidade dos sintomas depressivos segundo as escalas psicométricas para participantes com e sem diagnóstico prévio. Poços de Caldas/MG, Brasil, 2020.

\begin{tabular}{l|ccc|c}
\hline \multicolumn{1}{c|}{ Escalas } & Total & $\begin{array}{c}\text { Presença de sintomas } \\
\text { depressivos prévios }\end{array}$ & $\begin{array}{c}\text { Ausência de sintomas } \\
\text { depressivos prévios }\end{array}$ & p-value \\
\hline IDB & $37(50,7 \%)$ & $34(54,8 \%)$ & $03(27,3 \%)$ & 0,106 \\
Leve & $27(37,0 \%)$ & $22(35,5 \%)$ & $05(45,5 \%)$ & $03(27,3 \%)$ \\
Moderada & $09(12,3 \%)$ & $06(9,7 \%)$ & $03(27,3 \%)$ & $\mathbf{0 , 0 0 3}$ \\
Grave & $35(47,9 \%)$ & $32(51,6 \%)$ & $04(36,4 \%)$ & \\
Leve & $27(37,0 \%)$ & $23(37,1 \%)$ & $04(36,4 \%)$ & \\
Grave & $11(15,1 \%)$ & $07(11,3 \%)$ & $03(27,3 \%)$ & $\mathbf{0 , 0 0 3}$ \\
\hline MADRS & $35(47,9 \%)$ & $32(51,6 \%)$ & $04(36,4 \%)$ & \\
Leve & $27(37,0 \%)$ & $23(37,1 \%)$ & $04(36,4 \%)$ & \\
Moderada & $11(15,1 \%)$ & $07(11,3 \%)$ & & \\
\hline
\end{tabular}

Fonte: Dados da pesquisa Poços de Caldas (2020).

Os dados da Tabela 1 evidenciam que houve igualdade de intensidade do comportamento suicida em níveis leves nas três escalas de avaliação utilizadas, com mais de $50 \%$ dos participantes, enquanto o grupo com ausência de sintomas 
prévios apresentou percentuais maiores nos grupos Moderado e Grave. Tal evidencia se baseia na diferença entre os grupos que foi significativa ( $\mathrm{p}$-value $<0,003$ ) nos grupos moderados e graves com ausência de sintomas prévios. Em relação à presença de sintomas depressivos pela escala IDB, observamos dentre os que referiram ausência de sintomas depressivos prévios, 72,73\% apresentaram sintomas moderados e graves. Já para as escalas HAM-D e MADRS, 72,72\% dos que não apresentaram sintomas prévios, apresentaram sintomatologia moderada e grave. Os resultados obtidos pela aplicação das escalas psicométricas deste estudo evidenciam congruência entre as mesmas, com destaque para sintomatologia de intensidade moderada a grave.

Quanto ao histórico de doença em familiar dos participantes os dados evidenciaram significância estatística para o grupo com sintomatologia depressiva detectada via escalas para: doenças pré-existentes ( $\mathrm{p}$-value $<0,001$ ), presença de transtorno mental ( $\mathrm{p}$-value $<0,001$ ), uso de álcool/drogas, ( $\mathrm{p}$-value $<0,001$ ) e com grau de parentesco estatisticamente significativo para o grupo filhos ( $\mathrm{p}$-value $=0,022$ ). Quando observado o local do tratamento os dispositivos públicos de assistência à saúde se fizeram os mais citados, a exemplo da UBS e atenção secundária.

No Gráfico 1 constam dados quanto às características de histórico de doenças e transtorno mental dos participantes para com e sem presença de sintomas depressivos. 
Gráfico 1. Histórico de doença e transtorno mental dos participantes com e sem presença de sintomas depressivos. Poços de Caldas/MG, Brasil, 2020.
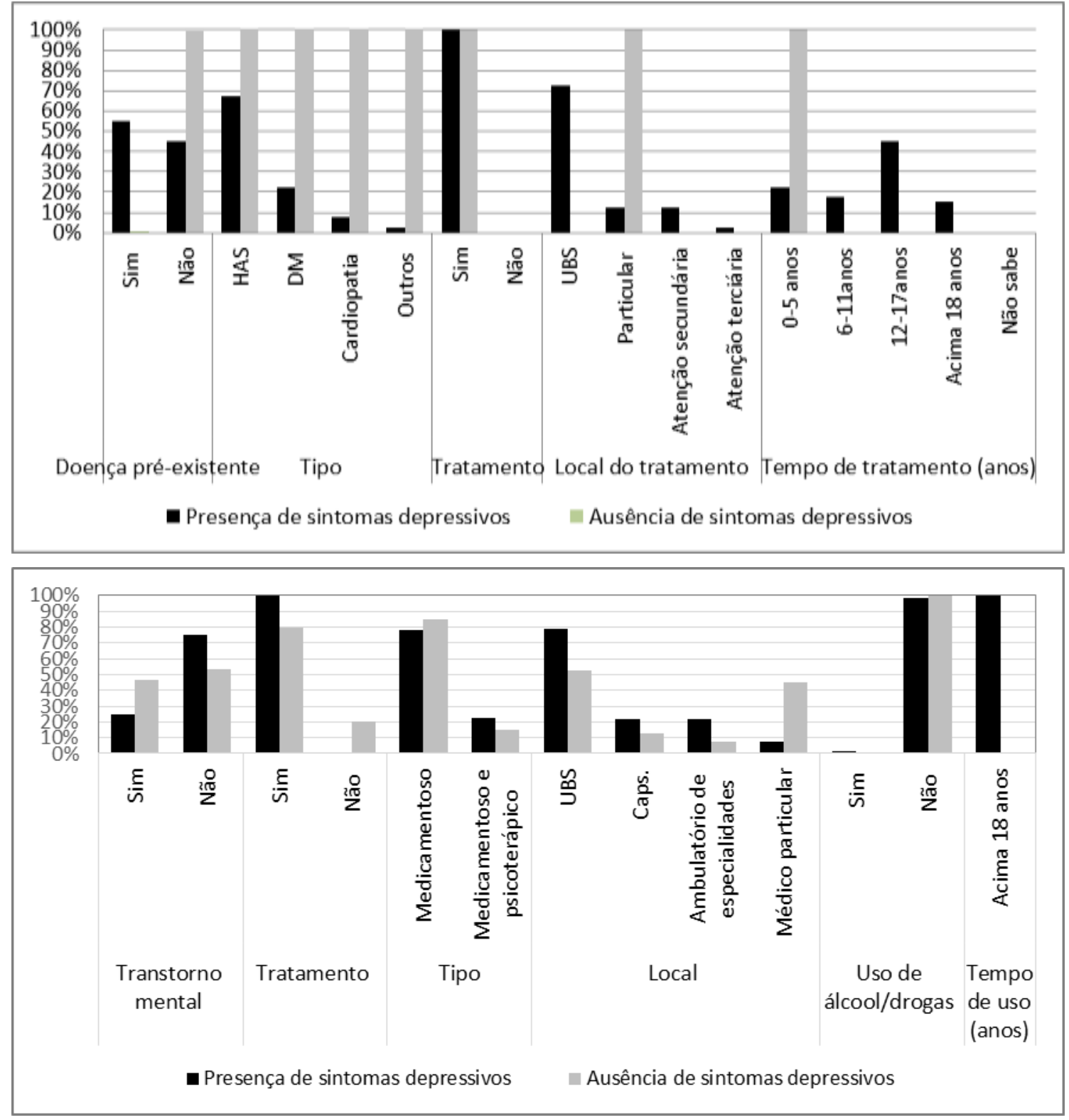

Fonte: Dados da pesquisa Poços de Caldas (2020).

Os resultados do gráfico acima demostram que no grupo com sintomas depressivos houve um percentual bem maior de doenças pré-existentes ( $54.8 \%$ contra $0.8 \%$ no grupo sem sintomas), com prevalência de $70 \%$ de Hipertensão Arterial Sistêmica (HAS), com 100\% das pessoas referindo tratamento prévio nos dois grupos. Quanto ao local do tratamento destas doenças prévias, $70 \%$ referiu tratamento em UBS no grupo com sintomas depressivos. Já o tempo de tratamento (anos) para as doenças pré-existentes foi de 45\% entre 12-17 anos no grupo com sintomas. Quanto ao transtorno mental, o grupo com sintomas depressivos apresentou percentual menor de transtorno mental ( $24.7 \%$ contra $46.5 \%$ no grupo sem sintomas). No que tange ao tratamento, $100 \%$ das pessoas com sintomas de transtorno mental referiram tratamento, sendo $83 \%$ do tipo medicamentoso, sem distinção de grupos com e sem sintomas. Os dispositivos públicos de saúde estiveram presentes em 96,9\% dos que necessitaram de tratamento para 0s transtornos mentais e apenas 1,4\% dos participantes referiram uso de álcool e por período acima de 18 anos. 
Verifica-se que os dados foram estatisticamente significativos para a questão da existência de hipertensão arterial sistêmica (HAS) e diabetes mellitus (DM) - (p-value < 0,001), com prevalência de $70 \%$ no grupo dos participantes com sintomas depressivos prévios. Quanto a ter transtorno mental, este foi referido por $77(38,50 \%)$ participantes (p-value $<0,001$ ), sendo que destes $62(80,51 \%)$ citaram depressão, $12(15,58 \%)$ ansiedade e $02(2,59 \%)$ transtorno bipolar, com maior concentração em tempo de tratamento entre 12-17 anos no grupo com sintomas prévios. Dos diagnosticados com depressão pelas escalas, $12(20,3 \%)$ relataram estar sem tratamento. Dos que referiram tratamento $54(83,1 \%)$ receberam tratamento exclusivamente medicamentoso; 25 (12,5\%) participantes com sintomas depressivos referiram ser atendidos em serviços públicos de saúde, principalmente na atenção básica.

Análise de doença familiar para a amostra como um todo e separadamente para os grupos com e sem presença de sintomas depressivos evidenciou-se estatisticamente significativa para presença de doença crônica degenerativa como diabetes mellitus e hipertensão arterial sistêmica, transtorno mental e uso de álcool e drogas.

Na Tabela 2 se encontra a distribuição dos participantes quanto ao diagnóstico prévio de depressão e tratamento para a amostra como um todo e em separado para os com e sem presença de sintomas depressivos, detectados via escalas psicométricas.

Tabela 2. Diagnóstico prévio de depressão e tratamento com e sem presença de sintomas depressivos. Poços de Caldas/MG, Brasil, 2020.

\begin{tabular}{|c|c|c|c|c|}
\hline Variáveis & Total & $\begin{array}{c}\text { Presença de } \\
\text { sintomas } \\
\text { depressivos }\end{array}$ & $\begin{array}{c}\text { Ausência de } \\
\text { sintomas } \\
\text { depressivos }\end{array}$ & p-value \\
\hline \multicolumn{5}{|l|}{ Diagnóstico depressão prévia } \\
\hline Sim & $62(31,0 \%)$ & $62(84,9 \%)$ & $0(0,0 \%)$ & $<0,001$ \\
\hline Não & $138(69,0 \%)$ & $11(15,1 \%)$ & $127(100,0 \%)$ & \\
\hline \multicolumn{5}{|l|}{ Tempo de diagnóstico (anos) } \\
\hline $0-5$ anos & $30(48,4 \%)$ & $30(48,4 \%)$ & ---- $(-)$ & \\
\hline 6-11anos & $13(21,0 \%)$ & $13(21,0 \%)$ & ---- $(-)$ & \\
\hline 12-17anos & $10(16,1 \%)$ & $10(16,1 \%)$ & ---- (-) & \\
\hline Acima 18 anos & $09(14,5 \%)$ & $09(14,5 \%)$ & $----(-)$ & \\
\hline \multicolumn{5}{|l|}{ Tratamento } \\
\hline $\operatorname{Sim}$ & $54(87,1 \%)$ & $54(87,1 \%)$ & ---- $(-)$ & \\
\hline Não & $08(12,9 \%)$ & $08(12,9 \%)$ & $----(-)$ & \\
\hline \multicolumn{5}{|l|}{ Local } \\
\hline UBS & $25(46,3 \%)$ & $25(46,3 \%)$ & ---- (一) & \\
\hline Caps. & $08(14,8 \%)$ & $08(14,8 \%)$ & $----(-)$ & \\
\hline Ambulatório de especialidades & $08(14,8 \%)$ & $08(14,8 \%)$ & ---- (-) & \\
\hline Médico particular & $13(24,1 \%)$ & $13(24,1 \%)$ & ---- (-) & \\
\hline \multicolumn{5}{|l|}{ Tempo de tratamento (anos) } \\
\hline $0-5$ anos & $36(66,7 \%)$ & $36(66,7 \%)$ & ---- (-) & \\
\hline 6-11anos & $05(9,3 \%)$ & $05(9,3 \%)$ & $----(-)$ & \\
\hline 12-17anos & $10(18,5 \%)$ & $10(18,5 \%)$ & ---- (-) & \\
\hline Acima 18 anos & $03(5,6 \%)$ & $03(5,6 \%)$ & ---- $(-)$ & \\
\hline
\end{tabular}


A Tabela 2 evidencia que $84.9 \%$ dos participantes com sintomas depressivos tiveram diagnóstico prévio de depressão. $\mathrm{O}$ tempo de diagnóstico (anos) apresentou maior prevalência entre 0 a 5 anos, seguido de 6 a 11 anos (ambos com 69.4\%). Já no que tange ao tratamento, $87,1 \%$ dos participantes referiram tratamento, sendo quase metade destes (46.3\%) em UBS e com tempo de tratamento (anos) com maior prevalência entre 0 a 5 anos (66,7\%).

Para a questão referente ao tempo de diagnóstico e tempo de tratamento surge uma discrepância para análise, pois $30(48,4 \%)$ participantes apresentaram tempo de diagnóstico de até 05 anos, porém $36(66,7 \%)$ dos que se trataram referiram tempo de tratamento em igual período, isso evidencia que $06(8,21 \%)$ participantes iniciaram tratamento antes de serem diagnosticados com transtorno depressivo.

Quando analisada a presença de sintomatologia depressiva por regiões geográficas do município de Poços de Caldas/MG, pode-se observar que a região Sul apresentou porcentagens mais significativas ( $\mathrm{p}$-value $=0.096$ ), sendo responsável por 23 (31,5\%) participantes. A região sul é a de maior vulnerabilidade social no município em questão.

No Gráfico 2 apresenta-se a distribuição dos participantes com sintomas depressivos e antecedentes prévios e tratamento para comportamento suicida.

Gráfico 2. Antecedentes prévios e tratamento para comportamento suicida dos participantes com presença de sintomas depressivos. Poços de Caldas/MG, Brasil, 2019.

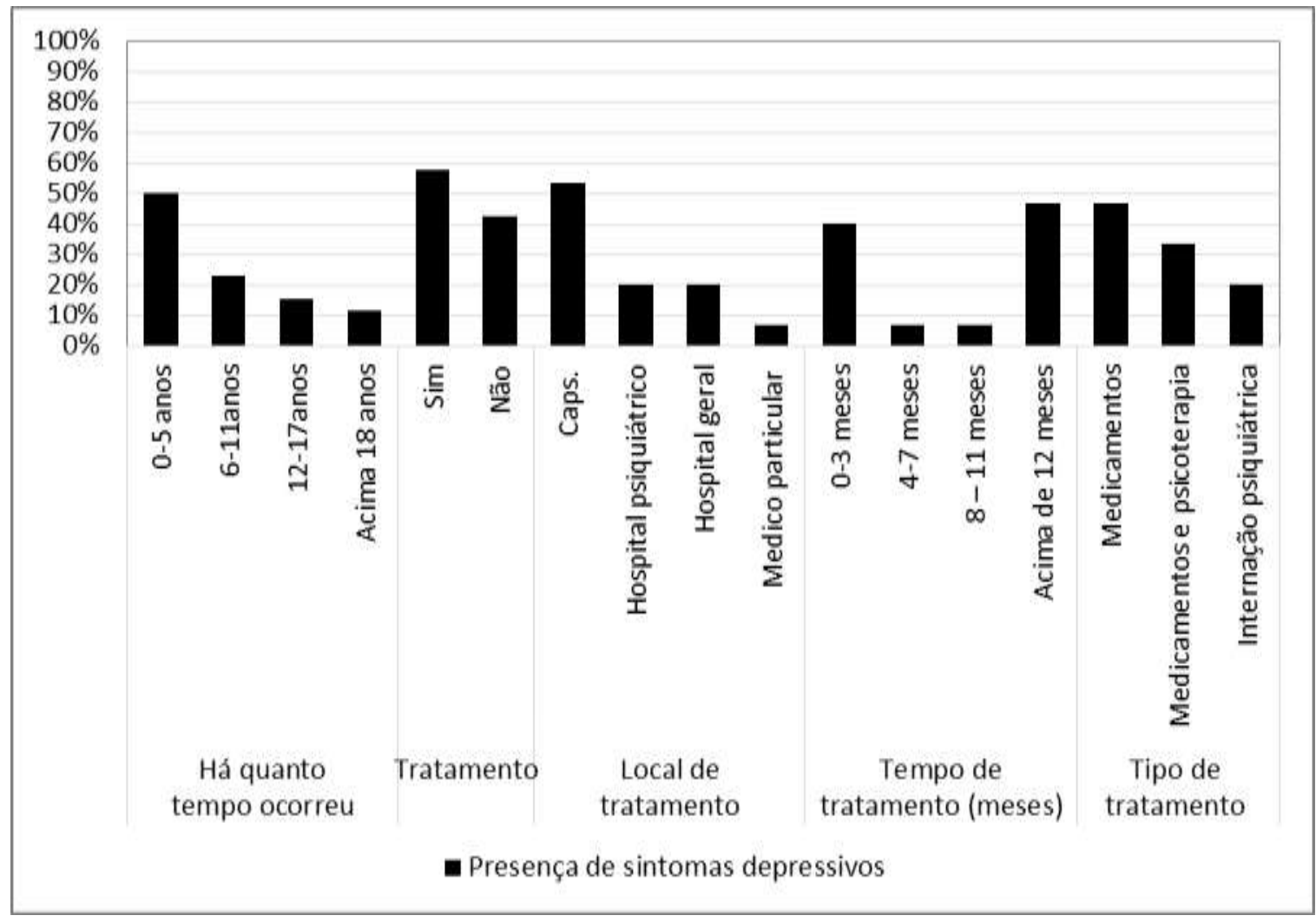

Fonte: Dados da pesquisa Poços de Caldas (2020).

Quanto ao comportamento suicida, o Gráfico 2 aponta que 35.6\% dos participantes com sintomas depressivos referiram ideação ou tentativa suicídio prévia, sendo destes $50 \%$ em período que variou de 0 a 5 anos e somente $42,3 \%$ destes não referiram tratamento para tal. O local de tratamento elencado por $53.3 \%$ dos participantes com ideação suicida foi o Caps, com tempo de tratamento para $40 \%$ destes entre 0 a 3 meses e de $46,7 \%$ acima de 12 meses. Para outros $46,7 \%$ o tratamento 
foi exclusivamente medicamentoso e $20 \%$ dos participantes necessitaram de internações psiquiátricas para tratamento do comportamento suicida.

Verificou-se pelo Gráfico 2 que o comportamento suicida esteve presente em $26(35,6 \%)$ dos participantes (p-value $<0,001)$. Ressalta-se que todos que relataram comportamento suicida tiveram sintomatologia depressiva apontada pelas escalas. Há 11 (42,3\%) participantes com comportamento suicida que referiram não ter realizado qualquer tratamento para a ideação suicida ou tentativa de suicídio. Quanto ao tratamento, 15 (57,7\%) dos participantes relataram que o fizeram e 13 (50\%) nos últimos 5 anos, sendo $06(40 \%)$ por período de até 3 meses e 07 (46,7\%) por período acima de 12 meses. 13 $(46,7 \%)$ dos participantes em tratamento fizeram uso apenas de medicação e 03 (11,5\%), receberam tratamento para o mesmo em hospitais psiquiátricos.

\section{Discussão}

Obteve-se predominância do sexo feminino, sendo $28,5 \%$ pertencente ao grupo que relatou sintomas depressivos prévios e 32,5\% no grupo que não relatou. Nossos dados corroboram com dados da literatura internacional (Jha et al., 2019; Searle, Blashki, Kakuma \& Yang, 2019; WHO 2018; Stein et al., 2017; Bromet et al., 2011), cuja prevalência de depressão entre as mulheres na população geral encontra-se na proporção de $75 \%$ de mulheres e $25 \%$ de homens, dados estes que também vão ao encontro aos estudos brasileiros (Gonçalves et al., 2018; Lopes, Hellwig, Silva \& Menezes, 2016; Costa, 2015; Munhoz, 2012)

As faixas etárias com participantes mais acometidos por sintomas depressivos foram as que vão ao encontro as faixas etárias de maior acometimento do transtorno depressivo citada pela WHO (2014; 2019), que evidenciam ser estas na adolescência (10 a 18 anos), idade adulta ( 24 a 45 anos) e acima de 55 anos. Verificou-se neste estudo, que os participantes com cor da pele autodeclarada branca apresentaram maior prevalência de depressão quanto aos com cor da pele autodeclarada preta. Esse resultado contraria estudos que observaram maior prevalência de depressão nos indivíduos de cor da pele preta, em comparação com os de pele branca no Brasil (Smolen \& Araújo, 2017; Quadros, Quevedo, Gonçalves, Horta \& Motta, 2020).

Quanto a relação estado civil e depressão, esta esteve associada ao status "casado", a contrariar estudos que evidenciam a solteirice, separação e viuvez como fatores desencadeadores para depressão devido quadro de solidão associado, muitas vezes relacionado a perda de rede de apoio (WHO, 2019; Gonçalves et al.,2018; APA, 2015; Rancans, Vrublevska, Snikere, Koroleva \& Trapencieris, 2013).

Embora religião não apresentou significância estatística com sintomas depressivos na amostra em estudo, a religiosidade pode influenciar o modo como pessoas lidam com situações de estresse, sofrimento e problemas vitais, pode levar a um sentimento de culpa, dúvida e autocrítica, o que pode contribuir para a depressão (Bretanha, Facchini, Nunes, Munhoz \& Tomasi, 2015; Máximo, 2010; Moreira-Almeida, Lotufo Neto \& Koenig, 2006). Já para Gonçalves et al. (2018) e Nery, Cruz, Faustino \& Santos (2018), a religiosidade/ espiritualidade surge como um aspecto determinante da vida humana e que, geralmente, tem associação positiva com boa saúde mental.

No que tange aos dados referentes à paternidade/maternidade, este foi um fator para o aparecimento de sintomas depressivos (91.8\% contra $67.7 \%$ no grupo sem sintomas - p- value<0,001), o que é compreensível, visto que o peso da responsabilidade no provimento material, emocional e educacional de filhos é um encargo de longa duração com múltiplas implicações para a vida dos pais (Arrais, Araújo \& Schiavo, 2018; WHO, 2018), embora tais dados se contraponham a ideação de filhos como fatores de proteção contra sintomas depressivos, amplamente explicitados em estudos a exemplo dos desenvolvidos por Epifânio, Genna, De Luca, Roccella e La Grutta (2015), National Research Council (Us) and Institute of Medicine - US (2009); Alt e Benetti (2008) e Saraiva e Coutinho (2007). 
A relação depressão e desemprego se fez presente a corroborar com a literatura internacional (Ornek, Weinmann, Waibel \& Radon, 2020; Rönnblad et al., 2019; IPEA, 2018, International Bank for Reconstruction and Development, 2018; Bajwa, Gastaldo, Di Ruggiero \& Knorr. 2018) e nacional (Magalhães \& Gomes, 2018; Seligmann-Silva, 2015; Bendassolli Alves \& Torres, 2014; Munhoz, 2012) acerca da causalidade entre desemprego e renda e depressão, pois quando não se atende as necessidades materiais básicas tende-se ao surgimento dos sintomas depressivos. A frustração decorrente de não conseguir um emprego ou ter que sobreviver de subempregos abaixo de sua capacidade ou por não ter capacitação adequada, ou ainda que não supram suas necessidades materiais lança as bases para a baixa autoestima, complexo de inferioridade, sentimento de impotência, vitimização e ressentimento, sendo estes gatilhos importantes para o surgimento dos sintomas depressivos (Sicsu, 2019).

Outro fator a ser considerado são as condições do trabalho, que também contribuem se não adequadas, para o sofrimento psíquico. Há evidências de estudos de associação entre sintomatologia depressiva, excesso de trabalho e ambiente laboral psiquicamente desgastante, devido excesso de cobranças, colocando o trabalhador em risco para a depressão, pois são fontes comuns de estresse crônico, ao qual se somam o temor de perder o emprego e o esgotamento mental e físico do excesso de trabalho (Qiao, Lu, Yan, Yao \& Pei, 2019; Nery, Cruz, Faustino \& Santos, 2018; Corrêa \& Rodrigues, 2017). Dejours $(2004 ; 1998)$ coloca que as exigências do trabalho e da vida são uma ameaça ao próprio trabalhador e causa intensos riscos de sofrimento psíquico, uma vez que as relações de trabalho nas organizações, frequentemente, despojam o trabalhador de sua subjetividade, o torna excluído e faz do homem uma vítima do seu trabalho. Um dos mais cruéis golpes que o homem sofre com o trabalho é a frustração de suas expectativas inicial sobre o mesmo, à medida que a propaganda do mundo do trabalho promete felicidade e satisfação pessoal/material para o trabalhador; porém, quando lá adentra, o que se tem é infelicidade, traduzida por insatisfação pessoal e profissional do trabalhador, a desencadear o sofrimento psíquico nas organizações.

Para a relação protetiva demostrada neste estudo em relação à depressão e uso de redes sociais, dados semelhantes foram obtidos em estudo desenvolvido por Sabik, Falat e Magagnos (2020) e Hunt, Marx, Lipson e Jordan (2018) concluíram que o uso das mídias sociais diminui a solidão e a depressão e evidenciam que reduzir o tempo de uso nas mídias sociais para até 30 minutos diários é um fator protetor e eficaz para a redução de depressão e para a sensação de solidão.

Obteve-se que 36,5\% da amostra deste estudo apresentou sintomatologia depressiva pela aplicação das escalas de avaliação, resultado maior que para população geral mundial, com média ponderada de depressão entre 16 a 20\% (WHO, 2019) e para a população brasileira com média ponderada de depressão entre 5,8\% a 7,6\% (Brasil, 2020; WHO, 2019), o que representa algo em torno de 12 a quase 16 milhões de brasileiros vivendo com depressão. Para a população geral de Minas Gerais a média para auto relato de depressão divulgada pela Pesquisa Nacional por a Amostra de Domicílios foi de 11,1\% (Brasil, 2020). Pode-se ter como justificativas para a prevalência elevada de depressão na amostra estudada na cidade de Poços de Caldas, a maior parte dela ser composta por mulheres $(78,1 \%)$, acima de 50 anos $(32,5 \%)$, boa parte dos participantes estar em desemprego ou subemprego, como observado pela Organização Mundial da Saúde (WHO, 2018; 2014). Outros possíveis fatores não foram verificados.

Pesquisa realizada por Stein et al. (2017), que serviu de base para o relatório publicado pela WHO (2018) sobre a epidemiologia transnacional do transtorno depressivo maior, onde estudos sobre quadros depressivos moderados e graves foram realizados simultaneamente em países com alta renda (Bélgica, França, Alemanha, Israel, Itália, Japão, Holanda, Nova Zelândia, Espanha e EUA) e média e baixa renda (Brasil, Colômbia, Índia, China, Líbano, México, África do Sul e Ucrânia) evidenciaram que nos países de alta renda, $14,6 \%$ da população já referiu depressão maior, sendo 5,5\% nos últimos 12 meses. Nos países de baixa e média renda, estes números são de 11,1\% da população geral, sendo de 5,9\% nos últimos 12 meses. Chama atenção que nos últimos 12 meses no Brasil esta taxa foi de 10,4\%. Este estudo evidencia que para os países de baixa e média renda, tal prevalência se deu duas vezes maior em mulheres se comparada aos homens na faixa etária acima de 50 anos, 
atrelado a ser solteiro, divórcios, viuvez e desemprego, e quanto maior a ocorrência destes fatores de risco, maior a prevalência de depressão; em nosso estudo há maior índice de depressão em mulheres, na faixa etária de 29 a 39 anos e casadas. Porém há de se considerar que a WHO (2014; 2018), coloca que em população acima de 50 anos o índice de depressão aumenta e nossa amostra apresenta $65(32,5 \%)$ do total geral dos participantes depressivos nesta faixa etária.

Quanto ao transtorno mental familiar, foram referidos ansiedade, depressão, e esquizofrenia, sendo estes dados corroborados por Senicato, Azevedo e Barros (2018), em estudo de base populacional. Outros autores (WHO 2018; Stein et al., 2017; WHO, 2014) também apontam que ter familiar com transtorno mental é fator de risco para surgimento de transtorno mental nos demais membros da família.

Quando questionados sobre familiar usuário de drogas, os dados nos remetem a um convívio desgastante, a servir de gatilho para quadros depressivos como encontrado em outros estudos (Quadros, Quevedo, Gonçalves, Horta \& Motta; WHO, 2018; Senicato, Azevedo \& Barros, 2018). Quanto ao tipo de droga utilizado, o álcool e crack foram predominantes, ao encontro de dados da Organização Mundial de Saude (WHO, 2018; WHO, 2014) sobre ser o álcool e a nicotina as drogas de maior consumo mundial e do $3^{\circ}$. Levantamento Nacional sobre o uso de drogas pela população brasileira, com o álcool a de maior consumo no Brasil (Bastos et al., 2017).

Para as doenças crônicas em familiar próximo, a exemplo da diabetes mellitus e hipertensão arterial, estas são consideradas como importante fator de risco para a depressão, devido ao desgaste emocional que acarretam ao longo de um tempo de cuidados, que pode durar de meses a muitos anos (Malta, Szwarcwald, \& Silva Júnior, 2019; WHO, 2018).

Dos diagnosticados com depressão pelas escalas, 12 (20,3\%) relataram estar sem tratamento, sendo tal achado inadmissível, pois pessoas deprimidas e sem tratamento apresentam maiores índices de sofrimento psíquico e somático, discriminação, isolamento social, interrupção ou diminuição do rendimento dos estudos e trabalho, abuso de álcool e outras drogas e aumento da mortalidade por suicídio e homicídio (WHO, 2018; Bonafé, Carvalho \& Campos, 2016; APA, 2015; WHO, 2014, Gascon et al., 2012).

A maioria dos participantes que recebeu tratamento foi exclusivamente medicamentoso, ao contrário aos pressupostos da Reforma Psiquiátrica brasileira a preconizar tratamento multiprofissional com reabilitação psicossocial, psicoterapia e práticas integrativas e complementares no tratamento efetivo dos transtornos mentais (OPAS, 2018; Brasil, 2018; Fleck et al., 2009). Por sua vez, há insuficiência quanto aos serviços em saúde mental, uma vez que a cidade onde o estudo foi realizado conta apenas com um CAPS tipo II para o tratamento de toda a população em sofrimento psíquico. Tal ineficiência das políticas púbicas de saúde não trata o problema relacionado aos transtornos mentais de forma eficiente e respeito a magnitude que o mesmo representa para o indivíduo, família e a própria saúde coletiva.

Quanto aos participantes com quadro de depressão moderada e grave e desconheciam tal fato, vem demonstrar o desconhecimento dos sintomas associados à depressão, a indicar para adaptação ao sofrimento e cronificação. Bonafé, Carvalho e Campos (2016) colocam ainda que as atitudes e crenças da pessoa deprimida em relação aos sintomas depressivos e ao tratamento se relacionaram fortemente à adesão/não-adesão ao tratamento, a exemplo da presença de efeitos colaterais associados a medicação, tempo e complexidade do tratamento e estigma relacionado ao transtorno.

Isto remete a importância do rastreio de depressão em população geral; além de nos remeter a ideia de que a informação ao público geral sobre os sinais e sintomas que caracterizam a depressão é uma ferramenta de prevenção importante, pois o conhecimento dos sintomas iniciais do estado depressivo pode levar o paciente e seus familiares a buscar ajuda profissional, antes do agravamento do transtorno (van Kleef, Bockting, van Valen, Aleman, Marsman \& van Tol, 2019; Rufino, Freschi \& Oliveira 2018) e evitar desnecessário sofrimento do indivíduo e diminuir os custos diretos e indiretos dos transtornos afetivos para o indivíduo e família (van Kleef, Bockting, van Valen, Aleman, Marsman \& van Tol, 2019).

Para a questão referente ao tempo de diagnóstico e tempo de tratamento surge uma discrepância na análise e outros 
estudos obtiveram discrepâncias parecidas e atribuíram tal fato a automedicação na depressão, que não acontece só por falta de um bom serviço público de saúde e com resolutividade, mas também pelo próprio hábito das pessoas deprimidas primeiramente recorrerem à automedicação. A busca por ajuda especializada ocorre quando a automedicação não surte efeito ou quando não se consegue comprar os medicamentos utilizados por conta própria devido ausência de receita médica (Annequin, Weill, Thomas \& Chaix, 2015; Bet, Penninx, Van Laer, Hoogendijk \& Hugtenburg, 2015).

Quanto ao comportamento suicida, este quando não é tratado ou tratado de forma ineficiente ou por tempo inadequado aumenta significativamente o risco de suicídio, sendo o histórico prévio de comportamento suicida o mais importante preditor de morte por suicídio na população geral (WHO, 2019; APA, 2015, Marcolan, 2018). Houve relação estatística na amostra para comportamento suicida.

Desta forma deve-se atentar para a ausência de atenção ao tema no Brasil, que se faz presente ao evidenciarmos que 08 participantes com ideação suicida não referiram tratamento, e dos que referiram tratamento, $40 \%$ o realizou nos últimos 3 meses, a apontar para o risco a que estes sujeitos correm de se matar. Desta forma, há necessidade de abordagem de tratamento multidimensional, como aponta a Organização Mundial de Saúde (WHO, 2019; WHO, 2014), a trabalhar a integralidade da assistência, a reabilitação psicossocial e práticas integrativas e complementares no tratamento efetivo do comportamento suicida.

Há de se considerar ainda a necessidade de políticas públicas que gerem efetivas mudanças sociais, e que tais mudanças estão atreladas a educação, vontade política e estratégia social, por meio da coordenação e colaboração entre múltiplos setores da sociedade, como saúde, educação, trabalho, agricultura, negócios, justiça, direito, defesa, política e mídia, a fim de conduzir a melhores resultados sobre esta questão tão complexa e que somente por meio de tais intervenções haverá acompanhamento efetivo destes indivíduos (Marcolan, 2018; WHO, 2019; 2014).

Quanto às limitações deste estudo obteve-se amostra pequena para análise quantitativa, uma vez que boa parte dos dados apresentou pouca significância estatística e o fato de ter sido realizado em única cidade que recebe usuários de cidades do entorno.

Como contribuição deste nosso estudo, os dados trazem pela primeira vez o panorama da sintomatologia depressiva em população geral de Poços de Caldas/MG, maior cidade do sul do estado de Minas Gerais, o que permitirá aos gestores municipais a realização de planejamento para o enfrentamento das questões abordadas.

\section{Conclusão}

Conclui-se que a presença de sintomatologia depressiva na população geral do município foi maior que a média mundial, nacional e estadual, com prevalência de intensidade dos sintomas moderado/grave. Participantes sem sintomatologia prévia apresentaram maior severidade dos sintomas depressivos. Relação entre depressão e suicídio se fez presente em mulheres, com filhos, desempregadas, baixa escolaridade, baixa renda, com familiar usuário de álcool/drogas, principalmente parentes de convívio próximo como filhos/pais e com doenças crônicas e transtornos mental; assim como o próprio participante ter transtorno mental, em especial depressão, e ter doenças crônicas como diabetes e hipertensão arterial. Quanto às sugestões para novos estudos destaca-se a necessidade de um estudo mais amplo, que contemple as várias cidades do entorno da cidade em questão, a fim de estabelecer maior complementaridade entre as estruturas e serviços de saúde mental que formam essas cidades. 


\section{Agradecimentos}

O presente trabalho foi realizado com o apoio da Coordenação de Aperfeiçoamento de Pessoal de Nível Superior Brasil (CAPES)

\section{Referências}

Alt, M. dos S., \& Benetti, S. P. da C. (2008). Maternidade e depressão: impacto na trajetória de desenvolvimento. Psicologia em Estudo. 13(2), $389-394$. https://doi.org/10.1590/S1413-73722008000200022

Annequin, M., Weill, A., Thomas, F., \& Chaix, B. (2015). Environmental and individual characteristics associated with depressive disorders and mental health care use. Annals of Epidemiology. 25(8), 605-612. 10.1016/j.annepidem.2015.02.002;

American Psychiatric Association (APA). (2015). Diagnostic an statistical manual of mental disorder, Fifth Editon (DSM - V). Washington: DC.

Arrais, A. da R., Araujo, T. C. C. F. de, \& Schiavo, R. de A. (2018). Fatores de Risco e Proteção Associados à Depressão Pós-Parto no Pré-Natal Psicológico. Psicologia: Ciência e Profissão. 38(4), 711-729. https://doi.org/10.1590/1982-3703003342016

Bajwa, G. \& Di, R. K. (2018). The Health of Workers in the Global Gig Economy. Global Health. 14(1):124. 10.1186/s12992-018-0444-8

Bastos, F. I. P. M. et al. (Org.). (2017). III Levantamento Nacional sobre o uso de drogas pela população brasileira. Rio de Janeiro: FIOCRUZ/ICICT. https://www.arca.fiocruz.br/handle/icict/34614

Bendassolli, P. F., Alves, J. S. C., \& Torres, C. C. (2014). Inventário sobre significado do trabalho de profissionais de indústrias criativas. Avaliação Psicológica. 13(2), 177-186. http://www.pedrobendassolli.com/textos/avaliacaopsicologica.pdf

Bet, P. M., Penninx, B. W., van Laer, S. D., Hoogendijk, W. J., \& Hugtenburg, J. G. (2015). Current and remitted depression and anxiety disorders as risk factors for medication nonadherence. The Journal of clinical psychiatry. 76(9), e1114-e1121. https://doi.org/10.4088/JCP.14m09001.

Bonafé, Fernanda Salloume Sampaio, Carvalho, Jéssica de Souza, \& Campos, Juliana Alvares Duarte Bonini. (2016). Depressão, ansiedade e estresse e a relação com o consumo de medicamentos. Psicologia, Saúde \& Doenças. 17(2), 105-119. https://dx.doi.org/10.15309/16psd170201

Brasil. Ministério da Saúde. (2018). Saúde Brasil 2018: Uma análise da situação de saúde e das doenças e agravos crônicos: desafios e perspectivas. Brasília: Ministério da Saúde. https://bvsms.saude.gov.br/bvs/publicacoes/saude_brasil_2018_analise_situacao_saude_doencas_agravos_cro nicos_desafios_perspectivas.pdf

Brasil. Ministério as Saúde. (2020). Depressão no Brasil entre 2006 a 2015. Brasilia: Ministério da Saúde. https://www.saude.gov.br/images/pdf/2019/julho/17/2019-014-Publicacao-02-07.pdf

Bretanha, A. F., Facchini, L. A., Nunes, B. P., Munhoz, T. N., Tomasi, E., \& Thumé, E. (2015). Depressive symptoms in elderly living in areas covered by Primary Health Care Units in urban area of Bagé, RS. Revista brasileira de epidemiologia = Brazilian journal of epidemiology. 18(1), 1-12. https://doi.org/10.1590/1980-5497201500010001

Bromet, E., Andrade, L. H., Hwang, I., Sampson, N. A., Alonso, J., de Girolamo, G., de Graaf, R., Demyttenaere, K., Hu, C., Iwata, N., Karam, A. N., Kaur, J., Kostyuchenko, S., Lépine, J. P., Levinson, D., Matschinger, H., Mora, M. E., Browne, M. O., Posada-Villa, J., Viana, M. C., Kessler, R. C. (2011). Crossnational epidemiology of DSM-IV major depressive episode. BMC medicine. 9, 90. https://doi.org/10.1186/1741-7015-9-90

Costa, F. A. da. (2018). Mulher, Trabalho e Família: Os impactos do trabalho na subjetividade da mulher e em suas relações familiares. Pretextos - Revista da Graduação em Psicologia da PUC Minas. 3(6), 434 -452. http://periodicos.pucminas.br/index.php/pretextos/article/view/15986

Epifanio, M. S., Genna, V., De Luca, C., Roccella, M., \& La Grutta, S. (2015). Paternal and Maternal Transition to Parenthood: The Risk of Postpartum Depression and Parenting Stress. Pediatric reports. 7(2), 5872. https://doi.org/10.4081/pr.2015.5872

Fleck, Marcelo P., Berlim, Marcelo T., Lafer, Beny, Sougey, Everton Botelho, Porto, José Alberto Del, Brasil, Marco Antônio, Juruena, Mário Francisco, \& Hetem, Luis Alberto. (2009). Revisão das diretrizes da Associação Médica Brasileira para o tratamento da depressão (Versão integral). Brazilian Journal of Psychiatry. 31(Suppl. 1), S7-S17. https://doi.org/10.1590/S1516-44462009000500003

Gascón, M. R. P., et al (2012). Prevalence of depression and anxiety disorders in hospitalized patients at the dermatology clinical ward of a university hospital. Anais Brasileiros de Dermatologia. 87(3), 403-407. https://doi.org/10.1590/S0365-05962012000300008Gonçalves.

Gonçalves, A. M. C., et al (2018). Prevalência de depressão e fatores associados em mulheres atendidas pela Estratégia de Saúde da Família. Jornal Brasileiro de Psiquiatria. 67(2), 101-109. https://doi.org/10.1590/0047-2085000000192

Güngör, B. B., Akgül, A. İ., Taymur, İ., Demirci, H., \& İnel, A. (2020). Evaluation of Eating Attitudes, Anger and Impulsivity in Atypical and Non-Atypical Depression and Assessment of Comorbidity of Binge Eating. Psychiatria Danubina. 32(1), 105-114. https://doi.org/10.24869/psyd.2020.105.

Hunt, M. C., Marx, C. D., Lipson, R. M., \& Jordan, A. (2018). Social media use increases depression and loneliness. Arts, humanities, \& social sciences. 17(5),354-359. https://penntoday.upenn.edu/news/social-media-use-increases-depression-and-loneliness

The World Bank. (2018). International Bank for Reconstruction and Development. Management's discussion \& analysis and financial statementes. Annual Report. https://documents.worldbank.org/en/publication/documents-reports/documentdetail/473291538159094998/annual-report-2018-managementsdiscussion-and-analysis-and-financial-statements-fiscal-2018 
Instituto de Pesquisa Econômica Aplicada. (2018). Políticas Sociais: acompanhamento $e$ análise. Brasília: IPEA. https://www.ipea.gov.br/portal/index.php?option=com_content\&view=article\&id=31656:politicas-sociais-acompanhamento-e-analise-no-25$2018 \&$ catid $=185:$ disoc $\&$ directory $=1$

Jha, M. K., Grannemann, B. D., Trombello, J. M., Clark, E. W., Eidelman, S. L., Lawson, T., Greer, T. L., Rush, A. J., \& Trivedi, M. H. (2019). A structured approach to detecting and treating depression in primary care: VitalSign6 project. Annals of family medicine. 17(4), 326-335. https://doi.org/10.1370/afm.2418

Lopes, C. S., Hellwig, N., Silva, G. A. \& Menezes, P. R. (2016). Inequities in access to depression treatment: results of the Brazilian National Health Survey PNS. Int J Equity Health. 15:154. https://doi.org/10.1186/s12939-016-0446-1

Magalhães, C. \& Gomes, D. (2018). Constituição e feminismo entre gênero, raça e direito: das possibilidades de uma hermenêutica constitucional antiessencialista e decolonial. Rev História: Debates e Tendências. 18(3): 343 -65. 10.5335/hdtv.18n.3.8593

Malta, D. C., Szwarcwald, C. L. \& Silva Júnior, J. B. (2019). Primeiros resultados da análise do laboratório da Pesquisa Nacional de Saúde. Revista Brasileira de Epidemiologia. 22 (Suppl. 2), E190001.SUPL.2. https://doi.org/10.1590/1980-549720190001.supl.2

Marcolan, J. F. (2018). For a public policy of surveillance of suicidal behavior. Revista Brasileira de Enfermagem. 71, 2343-2347. 10.1590/0034-7167-20180256

Marcolan, J. F., \& Silva, D. A. (2019). O comportamento suicida na realidade brasileira: aspectos epidemiológicos e da política de prevenção. Revista M. Estudos sobre a morte, os mortos e o morrer. 4, 31-44. http://www.seer.unirio.br/index.php/revistam/article/view/9290/7954

Marconi, M. A.; \& Lakatos, E. M. (2005). Fundamentos de metodologia científica. (6a ed.), Atlas.

Máximo, G. C. (2010). Aspectos sociodemográficos da depressão e utilização de serviços de saúde no Brasil (Tese de Doutorado). Universidade Federal de Minas Gerais, Bolo Horizonte. https://repositorio.ufmg.br/handle/1843/AMSA-8BCKKK

Moreira-Almeida, A., Lotufo Neto, F., \& Koenig, H. G. (2006). Religiousness and mental health: a review. Brazilian Journal of Psychiatry. 28(3), 242-250. Epub August 10, 2006. https://doi.org/10.1590/S1516-44462006005000006

Munhoz, T. N. (2012). Prevalência e fatores associados a depressão em adultos: estudo de base populacional. (Tese de Doutorado). Universidade Federal de Pelotas, Rio Grande do Sul. http://www.epidemio-ufpel.org.br/uploads/teses/Disserta\%20Roberta\%20Hirschmann\%20pdf.pdf

National Research Council (Us) and Institute of Medicine. (2009). Depression in Parents, Parenting, and Children: Opportunities to Improve Identification, Treatment, and Prevention. National Research Council (US) and Institute of Medicine (US) Committee on Depression, Parenting Practices, and the Healthy Development of Children; England MJ, Sim LJ, editors.Washington (DC): National Academies Press (US). 10.1590/S1413-73722008000200022.

Organização Pan-Americana da Saúde. (2018). Determinantes Sociais e Riscos à Saúde, Doenças Crônicas Não Transmissíveis e Saúde Mental. Secretaria de Atenção à Saúde - Departamento de Atenção Básica - DAB. Relatório técnico 56 - Vigilância, Promoção e Prevenção em Saúde. Brasília: Ministério da Saúde. https://www.paho.org/bra/index.php?option=com_content\&view=article\&id=6304:opas-e-oim-assinam-acordo-para-melhorar-saude-de-70-milhoesde-migrantes-nas-americas\&Itemid $=839$

Nery, B. L. S., Cruz, K. C. T., Faustino, A. M., \& Santos, C. T. B. (2018). Vulnerabilidades, depressão e religiosidade em idosos internados em uma unidade de emergência. Revista Gaúcha de Enfermagem. 39, e2017-0184. https://dx.doi.org/10.1590/1983-1447.2018.2017-0184.

Nordt, C., Warnke, I., Seifritz, E., \& Kawohl, W. (2015). Modelling suicide and unemployment: a longitudinal analysis covering 63 countries, 2000-11. The lancet. Psychiatry. 2(3), 239-245. https://doi.org/10.1016/S2215-0366(14)00118-7.

Ornek, K. O., Weinmann, T., Waibel, J. \& Radon, K. (2020). Precarious employment and migrant workers' mental health: a protocol for a systematic review of observational studies. Systematic Reviews. 9: 50. 10.1186/s13643-020-01313-w

Quadros, L. C. M., Quevedo, L. A., Gonçalves, H. D., Horta, B. L. \& Motta J. V. S. (2020) Common Mental Disorders and Contemporary Factors: 1982 Birth Cohort. Rev. Bras. Enferm. 73(1): e20180162. 10.1590/0034-7167-2018-0162

Rancans, E., Vrublevska, J., Snikere, S., Koroleva, I., \& Trapencieris, M. (2014). The point prevalence of depression and associated sociodemographic correlates in the general population of Latvia. Journal of affective disorders. 156, 104-110. https://doi.org/10.1016/j.jad.2013.11.022

Ornek, O. K., Weinmann, T., Waibel, J. et al. (2020). Precarious employment and migrant workers' mental health: a protocol for a systematic review of observational studies. Syst Rev. 9, 50. https://doi.org/10.1186/s13643-020-01313-w

Rufino, L., Freschi, V. \& Oliveira, M. F. (2018). Aspectos gerais, sintomas e diagnóstico da depressão. Revista Saúde em Foco, 10. http://portal.unisepe.com.br/unifia/wp-content/uploads/sites/10001/2018/11/095_ASPECTOS-GERAIS-SINTOMAS-E-DIAGN\%C3\%93STICO-DA-

DEPRESS\%C3\%83O.pdf

Sabik, N. J., Falat, J. \& Magagnos, J. (2020) When Self-Worth Depends on Social Media Feedback: Associations with Psychological Well-Being. Sex Roles. 82(7-8):411. 10.1007/s11199-019-01062-8

Saraiva, E. R. A. \& Coutinho, M. P. L. (2007). A estrutura das representações sociais de mães puérperas acerca da depressão pós-parto Psico-US. 12(2): 31926. https://www.scielo.br/pdf/pusf/v12n2/v12n2a20.pdf

Searle, K., Blashki, G., Kakuma, R., Yang, H., Zhao, Y., \& Minas, H. (2019). Current needs for the improved management of depressive disorder in community healthcare centres, Shenzhen, China: a view from primary care medical leaders. International journal of mental health systems. 13 , 47. https://doi.org/10.1186/s13033-019-0300-0.

Seligmann-Silva E. (2015). Unemployment and mental exhaustion - a challenge to public policies and trade unions. Labour Sciences Jornal. 4. https://www.ilo.org/wcmsp5/groups/public/---ed_dialogue/---actrav/documents/publication/wcms_731147.pdf 
Research, Society and Development, v. 10, n. 2, e26010212521, 2021

(CC BY 4.0) | ISSN 2525-3409 | DOI: http://dx.doi.org/10.33448/rsd-v10i2.12521

Senicato, C., Azevedo, R. C. S., \& Barros, M. B. A. (2018). Transtorno mental comum em mulheres adultas: identificando os segmentos mais vulneráveis. Ciência \& Saúde Coletiva. 23(8), 2543-2554. https://doi.org/10.1590/1413-81232018238.13652016.

Sicsu J. (2019). Brasil: é uma depressão, não foi apenas uma recessão. Revista de Economia Contemporâne. 23(1), e192312. 10.1590/198055272312

Smolen, J. R., \& Araújo, E. M. (2017). Raça/cor da pele e transtornos mentais no Brasil: uma revisão sistemática. Ciência \& Saúde Coletiva. 22(12), 40214030. https://doi.org/10.1590/1413-812320172212.19782016.

Stein, D. J., Lim, C., Roest, A. M., de Jonge, P., Aguilar-Gaxiola, S., Al-Hamzawi, A., Alonso, J., Benjet, C., Bromet, E. J., Bruffaerts, R., de Girolamo, G., Florescu, S., Gureje, O., Haro, J. M., Harris, M. G., He, Y., Hinkov, H., Horiguchi, I., Hu, C., Karam, A., ... WHO World Mental Health Survey Collaborators (2017). The cross-national epidemiology of social anxiety disorder: Data from the World Mental Health Survey Initiative. BMC medicine. 15(1), 143. https://doi.org/10.1186/s12916-017-0889-2.

van Kleef, R. S., Bockting, C., van Valen, E., Aleman, A., Marsman, J. C., \& van Tol, M. J. (2019). Neurocognitive working mechanisms of the prevention of relapse in remitted recurrent depression (NEWPRIDE): protocol of a randomized controlled neuroimaging trial of preventive cognitive therapy. BMC psychiatry. 19(1), 409. https://doi.org/10.1186/s12888-019-2384-0.

World Health Organization. (2014). Preventing Suicide - A Global Imperative. https://www.who.int/mental_health/suicide-prevention/world_report_2014/en/

World Health Organization. (2018). Mental Health ATLAS 2017. 2018. https://apps.who.int/iris/bitstream/handle/10665/272735/9789241514019eng.pdf?ua $=1$

World Health Organization. (2019). Suicide in the world: Global Health Estimates: https://apps.who.int/iris/bitstream/handle/10665/326948/WHO-MSDMER-19.3-eng.pdf?ua=1 